crystal growth. This assertion is supported by short ion-dipole distances in the product crystal, as well as the observation that the degree of polar order in the crystal varied directly with the magnitude of the dipole moment of the guest.

The scientists said that the polar order of the bulk crystal cannot be the result of guest-to-guest interactions because of the large separation between the guests brought about by the host structure. The guest dipoles are further separated by the tilting of the BPDS pillars and guest dipoles relative to the crystallographic c-axis of the crystal. This tilting is the result of the distortion of guanidinium sheets during crystal growth to accommodate the guest molecules. The value of the tilt angle is the same for all pillars and guests in a single layer of the crystal, but is reversed in adjacent layers, which serves to further separate the guest dipoles. In addition to the physical separation of the guests, the charged guanidinium sheets also act as a dielectric screen for dipole-dipole interactions between guests in adjacent layers. Additional evidence for the absence of guest dipoledipole interactions comes from molecular modeling, which shows that guest dipoledipole interactions favor a different guestdipole configuration than the one observed in the product crystal.

GREGORY KHITROV

\section{Electrodeposition Found as a Versatile Tool in the Preparation of Magnetic Nanostructures}

In an effort to investigate the suitability of electrodeposition for the controlled preparation of high-quality structures on an atomic scale, Yukimi Jyoko of Fukui National College of Technology in Japan and Walther Schwarzacher of the University of Bristol studied the growth of magnetic $\mathrm{Co} / \mathrm{Pt}$ and $\mathrm{CoNi} / \mathrm{Pt}$ nanostructures, as reported in the August issue of Electrochemical and Solid-State Letters. The obtained multilayered nanostructures exhibit perpendicular magnetic anisotropy and a large magnetic coercivity. The codeposition of $\mathrm{Ni}$, as well as the deposition overpotential, has a significant influence on the film properties.

\section{SBIR Update}

Ion Optics (Waltham, Massachusetts) has been awarded a Phase II Small Business Innovation Research (SBIR) contract from the National Science Foundation to develop a labquality water-vapor sensor, built on a single integrated circuit using microelectromechanical systems (MEMS) technology.
Magnetic multilayered nanostructures have received considerable attention since the discovery of perpendicular magnetic anisotropy, the magneto-optical Kerr effect, and giant magnetoresistance in metallic multilayers. New magnetic nanostructures have potential for application in high-density magneto-optical recording media or magnetoresistive sensor devices. "Most magnetic nanostructures have been fabricated by sputtering or molecularbeam epitaxy," said Schwarzacher, lecturer of physics. "Electrodeposition, however, may be more promising for all practical purposes, given the efficiency and simplicity of the required equipment."

Recently, he and Jyoko's group at Fukui proved that there is compositional modulation across successive layers in a $\mathrm{Co} / \mathrm{Pt}$ nanometer-multilayered structure grown on a $\mathrm{Pt}(111)$ substrate by electrodeposition under potential control. The present work investigates the role that codeposition of Ni plays on the films' properties.

The researchers grew multilayered $\mathrm{Co} / \mathrm{Pt}$ and $\mathrm{CoNi} / \mathrm{Pt}$ thin films on $\mathrm{Pt}(111)$ and $\mathrm{Cu}(111)$ single-crystal substrates by alternating deposition from a $\mathrm{CoSO}_{4}$ - and $\mathrm{NiSO}_{4}$-containing electrolyte and $\mathrm{H}_{2} \mathrm{PtCl}_{6}$ electrolyte under potential control. For comparison, alloy films were also prepared by electrodeposition under potential control from a single electrolyte of all three precursors. The CoNi/Pt films crystallized in a fcc structure, while the $\mathrm{Co} / \mathrm{Pt}$ films or alloy films showed a mixture of fcc Co, hcp Co, and Co stacking faults. X-ray diffraction superlattice profiles confirmed that the $\mathrm{CoNi} / \mathrm{Pt}$ films exhibit well-defined interfaces between successive layers with compositional modulation on the nanometerlength scale, while the $\mathrm{Co} / \mathrm{Pt}$ films do not contain pure Pt layers, but a compositionally modulated multilayer structure with graded transitions between a PtCo alloy and Co. "The microstructure and magnetic properties of electrodeposited $\mathrm{Co} / \mathrm{Pt}$ multilayers depend strongly on the deposition overpotential and the growth mechanism," said Jyoko. The fcc Co/Pt films showed perpendicular magnetic anisotropy and large magnetic coercivity values. With decreasing thickness of the Co layers, remanent perpendicular magnetization was observed. The CoNi/Pt films exhibited even larger coercivities, up to $1.5 \mathrm{kOe}$. "This suggests the presence of a nonferromagnetic or weakly ferromagnetic Ptrich phase localized at grain boundaries or segregated grains in the $\mathrm{CoNi} / \mathrm{Pt}$ nanostructure, which would pin domain-wall motion and also inhibit exchange interactions among grains," according to the researchers' report. This could occur by means of a place exchange between pas- sive hydroxide adsorbates, $\mathrm{Ni}(\mathrm{OH})_{2 \mathrm{ad}}$ and $\mathrm{Pt}(\mathrm{OH})_{2 \mathrm{ad}}$, in the adsorbed intermediate.

The researchers believe that electrodeposition is a promising path for the preparation of magnetic multilayered nanostructures. As the properties of the films depend strongly on the multilayer growth mechanism, the researchers concluded that further studies on the growth kinetics and mechanism are necessary to achieve improved structural and magnetic qualities in the electrodeposited nanostructures.

CORA LIND

\section{Cobalt Impunities Cause Nonlinear C urrent-Voltage Behavior in Niobium-Doped $\mathrm{SrTiO}_{3}$ Bicrystals}

Studies of the interfacial phenomena occurring at grain boundaries in polycrystalline electroceramic components have attributed nonlinearity in currentvoltage $(I-V)$ behavior to two sources: the degree of coherency of the grain boundary and the formation of additional interface states (such as double Schottky barriers). In the June issue of the Journal of the American Ceramic Society, researchers Takahisa Yamamoto, Katsuro Hayashi, Yuichi Ikuhara, and Taketo Sakuma of the University of Tokyo reported the formation of potential barriers in highly coherent bicrystals due only to impurities at the boundary, thus supporting the additional-interface-states theory.

Four niobium-doped $\mathrm{SrTiO}_{3}$ single crystals of dimensions $10 \mathrm{~mm} \times 10 \mathrm{~mm} \times 3 \mathrm{~mm}$ were polished to a mirror state along the chosen $\{001\}$ contact planes (corresponding to the $10 \mathrm{~mm} \times 10 \mathrm{~mm}$ faces). Metallic cobalt was evaporated onto the contact plane of one of the crystals to provide a controlled concentration of impurities in one sample (the "cobalt-evaporated" sample); the other contact surfaces remained clean. Bicrystals were then fabricated by carefully aligning and hot-pressing pairs of the single crystals at $1400^{\circ} \mathrm{C}$ for $10 \mathrm{~h}$ under $0.4 \mathrm{MPa}$ pressure in air. After cooling, 1-mm-thick plates were machined from the samples perpendicular to the grain boundaries for current-voltage testing, high-resolution transmission electron microscopy (HRTEM), and energydispersive spectroscopy (EDS).

Selected-area diffraction patterns produced by HRTEM show highly coherent boundaries in both samples, with no secondary amorphous phases present in the cobalt-evaporated sample. EDS analysis of the cobalt-evaporated sample clearly demonstrates that the cobalt is dissolved and distributed in the grain boundary, allowing the observed coherency to 